\title{
THE DERIVATIVE OF BAZILEVIČ FUNCTIONS
}

\author{
R. R. LONDON AND D. K. THOMAS
}

(Communicated by Irwin Kra)

\begin{abstract}
For $\alpha>0$, let $B_{1}(\alpha)$ be the class of normalised analytic functions $f$ defined in the open unit disc $D$ such that $\operatorname{Re}(f(z) / z)^{\alpha-1} f^{\prime}(z)>0$ for $z \in D$. Sharp upper and lower bounds are obtained for $\left|z f^{\prime}(z) / f(z)\right|$ when $f \in B_{1}(\alpha)$.
\end{abstract}

1. Introduction. For $\alpha>0$, denote by $B(\alpha)$ the class of analytic Bazilevic functions defined in the unit disc $D$, with $f(0)=0$ and $f^{\prime}(0)=1$ (e.g. $\left.[2,8]\right)$ and by $B_{1}(\alpha)$ the subclass of $B(\alpha)$ for which

$$
\operatorname{Re} f^{\prime}(z)(f(z) / z)^{\alpha-1}>0
$$

for $z \in D[7]$. Clearly $B_{1}(1)=R$, the class of analytic functions satisfying $\operatorname{Re} f^{\prime}(z)>0$ in $D$ first studied by Alexander $[\mathbf{1}]$.

In [9], it was shown that for $f \in R$ and $z \in D$,

$$
\left|\frac{z f^{\prime}(z)}{f(z)}\right| \leq \frac{-K}{(1-|z|) \log (1-|z|)}
$$

where $K$ is an absolute constant. Recently, London [5] obtained the sharp upper bound and Gray and Ruscheweyh [4], the sharp upper and lower bounds for $\left|z f^{\prime}(z) / f(z)\right|$ when $f \in R$.

In this paper, we give sharp upper and lower bounds for the wider class $B_{1}(\alpha)$. This sharpens the upper bound estimate given by El-Ashwah and Thomas [3].

2. Results. Following Gray and Ruscheweyh (loc. cit), we begin by defining a slightly wider class of functions.

DEFINITION. For $\alpha>0$, denote by $B_{0}(\alpha)$ the class of function analytic in $D$ with $f(0)=0, f^{\prime}(0)=1$ and satisfying the condition

$$
\operatorname{Re} e^{i \phi} f^{\prime}(z)(f(z) / z)^{\alpha-1}>0
$$

for $z \in D$ and for some $\phi=\phi(f) \in \mathbf{R}$.

THEOREM. For $f \in B_{0}(\alpha)$ and $|z| \leq r<1$,

$$
\frac{1-r}{\alpha(1+r)} \int_{0}^{1} t^{\alpha-1} \frac{1-t r}{1+t r} d t \leq\left|\frac{z f^{\prime}(z)}{f(z)}\right| \leq \frac{1+r}{\alpha(1-r)} \int_{0}^{1} t^{\alpha-1} \frac{1+t r}{1-t r} d t .
$$

The left-hand and right-hand inequalities are sharp in $B_{0}(\alpha)$ for the function

$$
f_{0}(z)=z\left(\alpha \int_{0}^{1} t^{\alpha-1} \frac{1+t z}{1-t z} d t\right)^{1 / \alpha}
$$

at $z=-r$ and $z=r$ respectively.

We use the method of Gray and Ruscheweyh (loc. cit) and require the following lemma.

Received by the editors September 28, 1987.

1980 Mathematics Subject Classification (1985 Revision). Primary 30C45.

(C)1988 American Mathematical Society $0002-9939 / 88 \$ 1.00+\$ .25$ per page 
LEMMA. Let $F(z)=1-z^{\alpha} /\left(\alpha \int_{0}^{z} \varsigma^{\alpha-1} /(1-\varsigma) d \zeta\right)$ and $G(z)=(1-F(z)) /(1-z)$. Then $F$ and $G$ have nonnegative Taylor coefficients about $z=0$, and in particular for $|z| \leq r<1$,

$$
\begin{gathered}
|F(z)| \leq F(r)<\lim _{t \rightarrow 1} F(t)=1 \\
\left|F^{\prime}(z)\right| \leq F^{\prime}(r)
\end{gathered}
$$

and

$$
|G(z)| \leq G(r)
$$

PROOF. Let

$$
H(z)=F(z)-1=-z^{\alpha} /\left(\alpha \int_{0}^{z} \frac{\varsigma^{\alpha-1}}{1-\varsigma} d \varsigma\right)
$$

Then clearly

$$
(1-z)\left(z H^{\prime}(z)-\alpha H(z)\right)=\alpha H^{2}(z) .
$$

With $H(z)=\sum_{k=0}^{\infty} c_{k} z^{k},(5)$ implies that

$$
(k-\alpha) c_{k}=(k-1-\alpha) c_{k-1}+\alpha \sum_{j=0}^{k} c_{j} c_{k-j},
$$

where $c_{-1}=0$. Thus

$$
c_{0}=-1, \quad c_{1}=\frac{\alpha}{\alpha+1}, \quad c_{2}=\frac{\alpha}{(2+\alpha)(\alpha+1)^{2}}
$$

and for $k \geq 3$,

$$
(k+\alpha) c_{k}=\left(k+\frac{\alpha^{2}-2 \alpha-1}{\alpha+1}\right) c_{k-1}+b_{k},
$$

where

$$
b_{3}=0 \quad \text { and } \quad b_{k}=\alpha \sum_{j=2}^{k-2} c_{j} c_{k-j} \quad \text { for } k \geq 4 .
$$

Since $3+\left(\alpha^{2}-2 \alpha-1\right) /(\alpha+1)>0$ a simple induction argument using (6) and (7) shows that $c_{k}>0$ for $k \geq 1$. Thus the coefficients of $F$ are nonnegative and (2) and (3) follow. Finally, with $G(z)=\sum_{k=0}^{\infty} d_{k} z^{k}$, we have

$$
d_{k}=1-\sum_{j=1}^{k} c_{j}=1-\lim _{t \rightarrow 1} \sum_{j=1}^{k} c_{j} t^{j} \geq 1-\lim _{t \rightarrow 1} F(t)=0
$$

and (4) follows.

PROOF OF THE THEOREM. From (1), it follows that

$$
\frac{z f^{\prime}(z)}{f(z)}=\frac{h(z)}{\alpha z^{-\alpha} \int_{0}^{z} \varsigma^{\alpha-1} h(\zeta) d \zeta}=\frac{h(z)}{\alpha \int_{0}^{1} t^{\alpha-1} h(t z) d t}
$$

where $\operatorname{Re} e^{i \phi} h(z)>0$ for $z \in D$. It follows from the Duality Principle [6, Theorem 1.1, Corollary 1.1 and Theorem 1.6] that any value assumed by the right-hand side of (8) for some $z \in D$ is also assumed for this $z$ when $h$ is a function of the form 
$(1+x z) /(1+y z)$ where $|x|,|y|=1$. Clearly in obtaining upper and lower bounds for $\left|z f^{\prime}(z) / f(z)\right|$, we may take

$$
h(z)=\frac{1+x z}{1-z} \quad \text { for }|x|=1 .
$$

We first obtain the lower bound in the Theorem. Using (8) and (9), we write

$$
\begin{aligned}
\frac{f(z)}{z f^{\prime}(z)} & =\frac{\alpha}{z^{\alpha}} \frac{1-z}{1+x z} \int_{0}^{z} \varsigma^{\alpha-1} \frac{1+x \zeta}{1-\varsigma} d \varsigma \\
& =\alpha \int_{0}^{1} t^{\alpha-1} \frac{1-z}{1+x z} \cdot \frac{1+x t z}{1-t z} d t .
\end{aligned}
$$

Now for $0 \leq t \leq 1$ and $|z|<1$,

$$
\frac{1+t|z|}{1+|z|} \leq\left|\frac{1+t z}{1+z}\right| \leq \frac{1-t|z|}{1-|z|} .
$$

Thus

$$
\left|\frac{1+x t z}{1+x z} \frac{1-z}{1-t z}\right| \leq \frac{1-t|z|}{1-|z|} \frac{1+|z|}{1+t|z|}
$$

and so

$$
\left|\frac{f(z)}{z f^{\prime}(z)}\right| \leq \alpha \frac{1+r}{1-r} \int_{0}^{1} t^{\alpha-1} \frac{1-t r}{1+t r} d t
$$

which is the required lower bound.

For the upper bound, we use (9) together with $F$ as defined in the Lemma to write

$$
\begin{aligned}
\alpha \int_{0}^{z} \varsigma^{\alpha-1} h(\zeta) d \zeta & =\alpha \int_{0}^{z} \varsigma^{\alpha-1}\left(-x+\frac{x+1}{1-\zeta}\right) d \zeta \\
& =z^{\alpha} \frac{1+x F(z)}{1-F(z)} .
\end{aligned}
$$

Hence (8) and (9) give

$$
\frac{z f^{\prime}(z)}{f(z)}=G(z) \frac{1+x z}{1+x F(z)}
$$

where $G(z)=(1-F(z)) /(1-z)$. Since $(1+a z) /(1+b z)$ maps the closed unit disc onto the circle centre $(1-a \bar{b}) /\left(1-|b|^{2}\right)$, radius $|a-b| /\left(1-|b|^{2}\right)$ provided $|b|<1$, we deduce that

$$
\begin{aligned}
\left|\frac{z f^{\prime}(z)}{f(z)}\right| & \leq|G(z)| \frac{|z-F(z)|+|1-F(z) \bar{z}|}{1-|F(z)|^{2}} \\
& =\frac{|G(z)|}{1-|F(z)|^{2}}\left(r\left|1-\frac{F(z)}{z}\right|+\left|1-r^{2}+r^{2}\left(1-\frac{F(z)}{z}\right)\right|\right) \\
& \leq \frac{|G(z)|}{1-|F(z)|^{2}}\left(r(1+r)\left|1-\frac{F(z)}{z}\right|+\left(1-r^{2}\right)\right) \\
& =\frac{1+r}{1-|F(z)|^{2}}\left(\frac{r}{\alpha}\left|F^{\prime}(z)\right|+(1-r)|G(z)|\right)
\end{aligned}
$$

where we have used $F^{\prime}(z)=\alpha G(z)(1-F(z) / z)$. 
It now follows from the Lemma that the last expression is maximal for $z=r$ and so

$$
\begin{aligned}
\left|\frac{z f^{\prime}(z)}{f(z)}\right| & \leq \frac{(1+r) G(r)}{1+F(r)}=\frac{1+r}{1-r} \frac{1-F(r)}{1+F(r)} \\
& =\frac{1+r}{1-r}\left(-1+2 \alpha r^{-\alpha} \int_{0}^{r} \frac{\varsigma^{\alpha-1}}{1-\varsigma} d \varsigma\right)^{-1} \\
& =(1+r)\left(\alpha(1-r) \int_{0}^{1} t^{\alpha-1} \frac{1+t r}{1-t r} d t\right)^{-1}
\end{aligned}
$$

which completes the proof.

\section{REFERENCES}

1. J. W. Alexander, Functions which map the interior of the unit circle upon simple regions, Ann. of Math. (2) 17 (1915), 12-22.

2. I. E. Bazilevix, Über einen Fall der Intergrier-barkeit in der Quadratur der Gleichung von LöwnerKufarev, Math. Sb. 37 (1955), 471-476.

3. R. M. El-Ashwah and D. K. Thomas, On the derivative of some Bazilevic functions (to appear).

4. F. Gray and S. Ruscheweyh, An estimate for functions with positive real part (to appear).

5. R. R. London, On functions whose derivative has positive real part, Proc. Amer. Math. Soc. 103 (1988), 521-524.

6. S. Ruscheweyh, Convolutions in geometric function theory, Sem. Math. Sup., Univ. Montréal 83 (1982).

7. R. Singh, On Bazilevic functions, Proc. Amer. Math. Soc. 38 (1973), 261-271.

8. D. K. Thomas, On Bazilevic functions, Trans. Amer. Math. Soc. 132 (1968), 353-361.

9. 68-70.

DEPARTMENT OF MATHEMATICS AND COMPUTER SCIENCE, UNiVERSity College OF SWANSEA, SWANSEA SA2 8PP, WALES 